\title{
The Investors' Behavior towards the Relationship between Bitcoin, Litcoin, Dash Coins, and Gold: A Portfolio Modeling Approach
}

\author{
Asma Maghrebi, Fathi Abid \\ Department of Management, Faculty of Economics and Management, Sfax, Tunisia \\ Email: maghrebiasma632@gmail.com, fathi.abid@fsegs.usf.tn
}

How to cite this paper: Maghrebi, A. and Abid, F. (2021) The Investors' Behavior towards the Relationship between Bitcoin, Litcoin, Dash Coins, and Gold: A Portfolio Modeling Approach. Journal of Mathematical Finance, 11, 495-511.

https://doi.org/10.4236/jmf.2021.113028

Received: June 29, 2021

Accepted: August 16, 2021

Published: August 19, 2021

Copyright $\odot 2021$ by author(s) and Scientific Research Publishing Inc. This work is licensed under the Creative Commons Attribution International License (CC BY 4.0).

http://creativecommons.org/licenses/by/4.0/

(c) (i) Open Access

\begin{abstract}
This study considers a market-based economy that is composed of two asset classes: one is a digital, cryptocurrency, and the other is real, gold. We demonstrated that coins like (BTC, LTC, and DASH) can substitute a traditional safe haven "gold" in an intertemporal investment portfolio to become a new form of safe haven. The cryptocurrency follows a Jump-diffusion process. However, gold prices follow an Ornstein-Uhlenbek process to characterize the stochastic nature of the market. The stochastic optimal control approach, combined with the strategic asset allocation and the intertemporal utility theory, are used through the derivation of a Hamilton-Jacobi-Bellman (HJB) equation to determine an explicit solution of the optimal allocation problem for investors with CRRA utility function. We considered the Gamma Lévy process to solve the optimization problem. By using the secant method, we determined numerically the optimal percentage invested in the two asset classes at each time over the holding period. Our results showed that an investor can substitute gold by coins (BTC, LTC, DASH) from an investment portfolio perspective. Although Gold is supposed to be the traditional safe-haven asset, the digital currency seems to emerge as a new form of safe-haven value in a risky environment.
\end{abstract}

\section{Keywords}

Cryptocurrency, Stochastic Modeling, Optimal Control, Lévy Process

\section{Introduction}

It is commonly agreed that cryptocurrencies are very volatile and cannot be modeled like ordinary assets or indices. The diffusion process, which seems to correspond to the modeling of the yields of cryptocurrencies, is a Brownian geo- 
metric diffusion process with a jump and a drift adjusted by the risk premium. Gold, on the other hand, presents a return whose variations follow an OrnsteinUhlenbek process. We considered an economy where there are two types of assets. The first type is very risky, cryptocurrency. Whereas, the other type with low risk is represented by gold. The investment strategies will be organized between these two types of assets belonging to two completely different asset classes: one is digital and the other is real. Investors will define their optimal strategies based on how they perceive these two worlds, digital and real. The opposite is also true because one can, from optimal investment strategies, infer the perception of investors of these two types of assets.

In recent years, cryptocurrency has been a growing decentralized payment system. There are many types of alternative currencies such as Bitcoin, Litcoin, Dash, Ripple, Etherium, etc. Among these coins, Bitcoin is the most used digital currency with the largest market capitalization [1]. This study is limited to three types of cryptocurrency, namely Bitcoin (BTC), Litcoin (LTC), and Dash (DASH). Cryptocurrencies are an alternative to mainstream currencies and they are often considered as a part of an alternative economy. If some investors lose trust in mainstream currencies or the entire economy, they might resort to coins. This is one of the reasons why Bitcoin has sometimes been called digital Gold [2]. The spectacular development of Bitcoin over the past few years led to speculation among professionals that Bitcoin is the modern hedge or the safe haven. However, Bitcoin has attracted increasing attention from practitioners, scholars, regulators and media. This idea has been extending very quickly and recently government authorities have paid more attention to the Bitcoin's position in the financial system. Besides, Cryptocurrencies have become more attractive to investors because they are not directly exposed to the political forces behind the market turmoil. Also, they are decentralized and not dictated by a single government, a single entity. In addition, they are not subject to the rules of a Central Bank or a political leader. This isolated nature has helped the cryptocurrencies to reach recent multi-year highs as the turmoil of the trade war triggered a sharp drop in global equities. Simply put, traders took refuge in cryptocurrencies as they are not subject to the same forces like conventional currencies. That is why people who feel less trust in governments see it as an alternative, said Aries Wang, co-founder of Bibox. The design of Bitcoin is closely related to gold. This led to the idea that Bitcoin has characteristics that are similar to gold, such as being a store of value and a safe haven. As a result to this idea, we chose a portfolio which is composed of gold and Bitcoin as well as other coins that have the same property. On the other hand, given the extreme volatility of Bitcoin, investors may instead need a safe haven from Bitcoin. We hypothesized that gold is such a traditional safe haven, and we analyzed how coins provide a new form of safe haven. Added to that, we examined if a Bitcoin can replace gold in an investment portfolio. This idea was discussed by Irene Henriques and Perry Sadorsky on 2018 [3] but the methodology that was adopted in their paper did not provide a clear answer to this question. 
[4] analyzed and compared the conditional variance properties of bitcoin and gold. They found differences in their structure. Hence, they showed that bitcoin and gold have fundamentally different properties as assets and links with the stock markets. They concluded that bitcoin does not represent new gold. In this work, we will give another point of view justified by calculations and analyzes followed by interpretation which shows different conclusions. In fact, in this paper we consider a market economy made up of two asset classes: one is digital, cryptocurrency and the other is real, gold. It is shown that cryptocurrencies like (BTC, LTC and DASH) can replace a traditional safe haven "gold" in an intertemporal investment portfolio. Cryptocurrencies follow a leap diffusion process and gold prices follow an Ornstein-Uhlenbek process to characterize the stochastic nature of the market. The stochastic optimal control approach, combined with the optimal asset allocation strategy and the intertemporal utility theory, is used through the derivation of a Hamilton-Jacobi Bellman (HJB) equation to determine an explicit solution of the optimal allocation problem for investors with the CRRA utility function. We considered a gamma process to numerically determine, using the secant method, the optimal percentages invested in the two asset classes at any time during the holding period.

The present paper aims at answering the following questions: can coins (BTC, LTC, and DASH) replace gold in an investment portfolio? Can these coins represent a current and future safe-haven value according to their characteristics? The parameters of BTC, LTC, DASH, and gold are estimated using US data for the period 2014-2019.

In the upcoming section, a survey of the relevant literature will be exposed. However, in section 3 we focus on the model formulation of the dynamic portfolio selection problem. Section 4 is devoted to presenting the stochastic control problem solution within a power utility framework. As for Section 5 , it illustrates the optimal solution with a Gamma process. Section 6 presents the problem discretization, and the parameter estimates. The penultimate section serves to discuss the optimal portfolio choices and behavioral implications. Whereas, Section 8 encompasses the results' synthesis, comments, and the concluding remarks.

\section{Review of Past Work}

In recent decades, considerable attention has been paid to cryptocurrency. Investors are drawn to the potential for high returns, the benefits of diversification, the increase rates in market capitalization, volumes traded as well as the speed of transactions [5]. Despite the growing interest incryptocurrency as digital assets, the current literature on economics and finance still lacks empirical evidence on its diversification, coverage, and safe haven properties compared to other assets.

According to the market assets' views, cryptocurrencies are very volatile in their pricing compared to fiducial currency. The professionals who follow the stock charts have seen how the price of Bitcoin often fluctuates very violently, even at the level of ten percent. Indeed, unforeseen changes in market sentiment 
can result in large and sudden price movements. Generally speaking, this volatility is very frightening for investors and the markets, especially for unexperienced investors and speculators who speculate on the price of Bitcoin for short-term gain. This volatility is justified by several factors. One of the most certain causes of Bitcoin volatility is speculation. The cycle of buying and selling Bitcoins, often due to investor emotions, news, political or international decisions, creates sharp price fluctuations and generates high volatility. [6] [7] [8] and [9] recently showed that the volatility of cryptocurrencies is extreme. However, this volatility attracts the investors' attention because it provides more performance. [10] showed that Bitcoin's volatility is extreme and that prices fluctuate not only considerably over longer horizons, but also over short horizons. Bitcoin's volatility leads to a greater dispersion of returns and risk [11] [12] and [13]. Despite being very volatile, cryptocurrencies have become very profitable.

The more volatile is the financial value, the riskier it is, the more it will offer interesting trading opportunities. Therefore, price volatility allows investors to be able to make profits on the markets. Also, cryptocurrencies can offer significant trading opportunities. Indeed, the volatility of cryptocurrency increases the probability of gains. Bitcoin is very volatile and its inclusion in a diversified portfolio is very profitable [14]. [15] used tests and a traditional mean-variance framework to show that including a small portion of Bitcoin in a well-diversified portfolio can dramatically improve the trade-offs between risk and return. More recently, [13] applied a conditional value-at-risk framework and backtest portfolio valuation techniques to achieve similar results.

Traditionally, gold is the ultimate asset used as a safety net for economies, a reserve of currency and the most robust safe haven for investors. Recently, cryptocurrencies have sparked controversy as a new safe haven. Recent studies have found that Bitcoin may show signs of safe haven. Bitcoin is sometimes referred to as digital gold as Bitcoin is seen as part of an alternative economy and investors could resort to Bitcoin if they lose faith in current currencies [16] [17]. [18] have shown that Bitcoin is a new form of safe haven, and if a portfolio contains several assets at the same time, Bitcoin can play several roles, such as a safe haven, a hedge asset and a means of diversification. [19] showed that Bitcoin had many similarities to gold. [20] answered the question "to what extent does Bitcoin act as a hedge asset and a safe haven?" Using linear regression, [21] studied Bitcoin's ability as a safe haven and hedge against the S\&P 500 index and foreign exchange (FX) markets over different time periods. [22] used a dynamic conditional correlation model to examine whether Bitcoin can be used as a safe haven and hedge for bonds, gold, major global stock indices, oil, the general index of commodities, commodities and the US dollar index. They showed that Bitcoin has a safe haven and hedging properties that vary from horizon to horizon. You can use Bitcoin or other cryptos as a safe haven. Indeed, if we invest in cryptocurrencies, we will take greater risks than with company shares, the fluctuations being much greater but also we will generate greater returns [4].

Much previous research shows that Bitcoin can be used as a hedge, a safe- 
heaven, or a diversifier against traditional financial assets (e.g., stock, bond, commodity and USD) for investors. Our work further investigates in more detail the properties of cryptocurrencies as a safe haven. The strong point of this work is the right choice of model and the methodology adopted. Indeed, in the present study, the diffusion process, which seems to correspond to the modeling of the yields of cryptocurrencies, is a Brownian geometric diffusion process with a jump and a drift adjusted by the risk premium. Gold, on the other hand, presents a return whose variations follow an Ornstein-Uhlenbek process. The advantage of applying such an approach is to take into account the stochastic nature of the market, and the high volatility of cryptocurrency and the low volatility of Gold. This helps us a lot to find reliable results.

\section{Research Design and Methodology}

Let $(\Omega, F, P)$ be a complete probability space and $\left\{F_{t}\right\}_{t \geq 0}$ a filtration satisfying the usual conditions. We set up the portfolio selection model in a continuoustime framework. The two-asset model involves two different classes of assets: one is very risky (cryptocurrency) and the other is less risky (gold). So, we consider a financial market consisting of a cryptocurrency and gold. Let the Gold dynamics $B(t)$ be given by the Ornstein-Uhlenbeck process to model the evolution of the spot rate and, $P(t)$ be the price evolution of the cryptocurrency risky asset which is modeled by the process.

Accordingly, the dynamics of the gold spot rate of return and the prices of the coins are as follows:

$$
\begin{gathered}
\frac{\mathrm{d} B(t)}{B(t)}=r(t) \mathrm{d} t \\
\mathrm{~d} r(t)=\alpha[\beta-r(t)] \mathrm{d} t+\sigma_{r} \mathrm{~d} X^{r}(t)
\end{gathered}
$$

where parameters $\alpha, \beta$ and $\sigma_{r}$ are strictly positive constants and correspond to the degree of mean reversion, long-return mean, and volatility of the gold.

$$
\frac{\mathrm{d} P(t)}{P(t)}=\left[r(t)+\lambda \sigma_{p}\right] \mathrm{d} t+\sigma_{p} \mathrm{~d} X^{p}(t)+\int_{\Re} \gamma(t, z) \tilde{N}(\mathrm{~d} t, \mathrm{~d} z)
$$

We will suppose that $\gamma(t, z)=z$. To obtain a unique solution to this stochastic differential equation, we must have $1+\gamma(t, z)>0$ which means that $z>-1$, this implies that we may only suppose jump sizes higher than -1 . As we have jumps in both directions (positive and negative), but negative jumps are not too large, we assume that:

$$
\frac{\mathrm{d} P(t)}{P(t)}=\left[r(t)+\lambda \sigma_{p}\right] \mathrm{d} t+\sigma_{p} \mathrm{~d} X^{p}(t)+\int_{-1}^{\infty} z \tilde{N}(\mathrm{~d} t, \mathrm{~d} z)
$$

where: $\mu(t)=r(t)+\lambda \sigma_{p}$ with $\mathrm{d} \mu(t)=\mathrm{d} r(t)$ and $\tilde{N}$ is a martingale,

$\mathrm{d} X^{p}(t)$ and $\mathrm{d} X^{r}(t)$ are standard Brownian motions with

$\mathrm{d} X^{p}(t) \mathrm{d} X^{r}(t)=\rho \mathrm{d} t . \rho$ is the correlation between the random change in the gold spot rate and the random return on the coin asset. 
We assume that the instantaneous rate of gold is the short rate a $r(t)$ and the expected return $\mu(t)$ on the cryptocurrency equals the spot rate plus a risk premium. If $y(t)$ represents the optimal fraction of the wealth invested in coins, then $1-y(t)$ is the optimal fraction of the wealth invested in gold.

The dynamics of wealth $W(t)$ is given by:

$$
\mathrm{d} W(t)=y(t) W(t) \frac{\mathrm{d} P(t)}{P(t)}+(1-y(t)) W(t) \frac{\mathrm{d} B(t)}{B(t)}
$$

Substituting (1) and (2) into (4) and simplifying the equation, we have

$$
\mathrm{d} W(t)=\left[r(t)+y(t) \lambda \sigma_{p}\right] \mathrm{d} t+y(t) W(t) \sigma_{p} \mathrm{~d} X^{p}(t)+y(t) W(t) \int_{-1}^{\infty} z \tilde{N}(\mathrm{~d} t, \mathrm{~d} z)
$$

where $W(0)=W_{0}$ stands the initial wealth.

\section{The Stochastic Optimal Control Problem Solution}

Letting $U[W(t), t]$ be a concave, additively separable utility defined over wealth, and let's assume that the investor allocates his wealth between the two assets to maximize his expected utility of the terminal wealth. We suppose that the investor has a power utility function.

We obtain the maximized utility function $J[W(t), r, t]$ at the time $t \in[0, T]$ such that

$$
J[W(t), r, t]=\sup _{y} E_{t} \int_{t}^{T} U[W(s), s] \mathrm{d} s
$$

Subject to the continuous-time budget constraint of Equation (5). where, $U[w]=\frac{w^{1-\gamma}}{1-\gamma} \quad(0<\gamma<1)$ is the risk averseness parameter.

Using power utility has two advantages. The first advantage is that an explicit solution can be found for our portfolio selection problem with Power utility but not with other utility functions. The second advantage is that Power utility allows an optimal solution independent of wealth and thus it simplifies the derivation. [23] [24] showed that the utility of an investor is characterized by a decreasing absolute risk aversion and a constant relative risk aversion. These properties are compatible with the Power utility.

Equation (6) can be simplified to

$$
J[W(t), r, t]=\sup _{y} E_{t}\left\{\int_{t}^{T} J[W+\mathrm{d} W, r+\mathrm{d} r, t+\mathrm{d} t]\right\}
$$

We need to calculate the infinitesimal generator of an Ito-Lévy process (geometric Lévy), more specifically the generator of Ito-Lévy diffusion to find the Hamilton-Jacobi-Bellman equation. By simplifying the general version of the Ito process, assuming that $E[P(t)]<\infty$, we find the form that we will use throughout the paper. The infinitesimal generator for a Lévy process with jumps is found using the Ito-Lévy theorem with the fact that $\tilde{N}$ is a martingale. Letting $y(t) \in A$ and $W(t)$ the asset portfolio value process, the HJB equation asso- 
ciated with the dynamic portfolio problem is [25].

$$
\begin{aligned}
& \sup _{y}\left(J_{t}+J_{w}\left[r+y \lambda \sigma_{p}\right] W+J_{r} \alpha(\beta-r)+\frac{1}{2} J_{w w} W^{2} \sigma_{p}^{2} y^{2}+\frac{1}{2} J_{r r} \sigma_{r}^{2}\right. \\
& \left.+J_{r w} \sigma_{r} \sigma_{p} \rho y W+\int_{-1}^{\infty}\left[J(r, s, W(1+y z))-J(r, s, W)-J_{w} y W z\right] v(\mathrm{~d} z)\right)=0
\end{aligned}
$$

Here, $J_{t}, J_{W}, J_{r}, J_{W W}, J_{r W}$ and $J_{r r}$ denote the first and second order partial derivatives with respect to $t, r$ and $W$ in the normal way. To solve the HJB equation, let the value function $J(t, r, W)=W^{\gamma} f(t, r)$, where $f(T, r)=1$ for all $r$. We have $J_{t}=W^{\gamma} f_{t}, J_{W}=\gamma W^{\gamma-1} f, J_{r}=W^{\gamma} f_{r}, J_{r W}=\gamma W^{\gamma-1} f_{r}$, $J_{W W}=\gamma(\gamma-1) W^{\gamma-2} f, \quad J_{r r}=W^{\gamma} f_{r r}, J(s, r, W(1+y z))=W^{\gamma}(1+y z)^{\gamma} f$.

Now replacing $J$-related terms by $f$-related terms in Equation (8) and simplifying to obtain a second order PDE for $f$ which is written as follows:

$$
\begin{aligned}
& W^{\gamma} f_{t}+\gamma W^{\gamma-1} f r W+W^{\gamma} f_{r} \alpha(\beta-r)+\frac{1}{2} W^{\gamma} f_{r r} \sigma_{r}^{2} \\
& +\sup _{y}\left\{\gamma \lambda \sigma_{p} W W^{\gamma-1} y f+\frac{1}{2} \gamma(\gamma-1) W^{\gamma-2} W^{2} \sigma_{P}^{2} y^{2} f+\gamma W^{\gamma-1} W \sigma_{r} \sigma_{p} \rho y f_{r}\right. \\
& \left.+\int_{-1}^{\infty}\left[W^{\gamma}(1+y Z)^{\gamma} f-W^{\gamma} f-\gamma W^{\gamma-1} f W y z\right] v(\mathrm{~d} z)\right\}=0
\end{aligned}
$$

Dividing each side by $W^{\gamma}$, we obtain the following Hamilton-Jacobi-Bellman (HJB) equation.

$$
\begin{aligned}
& f_{t}+\gamma r f+\alpha(\beta-r) f_{r}+\frac{1}{2} f_{r r} \sigma_{r}^{2}+\sup _{y}\left\{\gamma \lambda \sigma_{p} y f+\frac{1}{2} \gamma(\gamma-1) f \sigma_{p}^{2} y^{2}\right. \\
& \left.+\gamma f_{r} \sigma_{r} \sigma_{p} \rho y+\int_{-1}^{\infty}\left[(1+y z)^{\gamma} f-f-\gamma f y z\right] v(\mathrm{~d} z)\right\}=0
\end{aligned}
$$

The term $W^{\gamma}$ is eliminated from the HJB equation because power utility is used.

By applying the first-order conditions, we obtain

$$
\left.\lambda \sigma_{p} f+(\gamma-1) f \sigma_{p}^{2} y+f_{r} \sigma_{r} \sigma_{p} \rho+f \int_{-1}^{\infty}\left[(1+y z)^{\gamma-1}-1\right] z v(\mathrm{~d} z)\right\}=0
$$

With: $y=y\left(f, f_{r}, v\right)$

Consequently, we conjecture a solution with the following form, $f(t, r)=g(t) \exp (A(t) r)$ with terminal conditions $g(T)=1, A(T)=0,[26]$.

The form of $f(t, r)$ is the solution of PDE, with $g$ and $A$ being regular functions.

The partial derivative with respect to $r$ is

$$
f_{r}=A(t) g(t) \exp (A(t) r) .
$$

After replacing $f$ and $f_{r}$ terms in (9) and simplifying the equation, we obtain

$$
\begin{aligned}
& \lambda \sigma_{p} g(t) \exp (A(t) r)+(\gamma-1) \sigma_{p}^{2} y g(t) \exp (A(t) r) \\
& +A(t) g(t) \exp (A(t) r) \sigma_{r} \sigma_{p} \rho \\
& \left.+g(t) \exp (A(t) r) \int_{-1}^{\infty}\left[(1+y z)^{\gamma-1}-1\right] z v(\mathrm{~d} z)\right\}=0
\end{aligned}
$$


Dividing by $g(t) \exp (A(t) r)$ :

$$
\left.\lambda \sigma_{p}+(\gamma-1) \sigma_{p}^{2} y+A(t) \sigma_{r} \sigma_{p} \rho+\int_{-1}^{\infty}\left[(1+y z)^{\gamma-1}-1\right] z v(\mathrm{~d} z)\right\}=0
$$

where: $A(t)=\frac{\gamma}{\alpha}[1-\exp (\alpha(T-t))]$

After replacing $A(t)$ in Equation (10), we obtain an optimal portfolio strategy for the given portfolio problem.

$$
\begin{aligned}
& \lambda \sigma_{p}+(\gamma-1) \sigma_{p}^{2} y+\sigma_{r} \sigma_{p} \rho \frac{\gamma}{\alpha}[1-\exp (\alpha(T-t))] \\
& -\int_{-1}^{\infty}\left[1-(1+y z)^{\gamma-1}\right] z v(\mathrm{~d} z)=0
\end{aligned}
$$

This equation is non-linear in $\mathrm{y}$ and the difficulty comes from the integral term. Theoretically, we can solve this equation through any Lévy measure. We can provide a numerical result for the portfolio selection problem studied. We can compare the value of the optimal portfolio weights via different Lévy measures. General Lévy processes may include a complicated Lévy measurement that makes it difficult to find an optimal or even an impossible solution. However, if we limit our study to the case where the Lévy measure is continuous for the Lebesgue measure, we can provide tangible results.

So we solve the Equation (11) to determine the proportion of risky investment

Theorem 1:

We call a Lévy measure a non-negative measure $v(\mathrm{dz})$ on $\mathfrak{R}$ satisfying $v(\{0\})=0$ and

$$
\int_{\Re}\left(Z^{2} \wedge 1\right) v(\mathrm{~d} z) \prec \infty
$$

Now, we can solve the problem of portfolio selection for an investor, who is confronted with different investment opportunities described above, with a finite time horizon.

\section{Optimal Solution with a $\Gamma(1,1)$ Process}

We consider the case of the $\Gamma(1,1)$-Lévy process whose Lévy measure is given by:

$$
v(\mathrm{~d} z)=z^{-1} \mathrm{e}^{-z} I(z>0)
$$

This is a Lévy measure since it satisfies the inverse of Lévy-Khintchine theorem for the existence of a Lévy process, it suffices to show that

$$
\int_{\Re} \min \left(1, z^{2}\right) v(\mathrm{~d} z)=\int_{0}^{\infty} \min \left(1, z^{2}\right) z^{-1} \mathrm{e}^{-z} \mathrm{~d} z=\int_{0}^{1} z \mathrm{e}^{-z} \mathrm{~d} z+\int_{1}^{\infty} z^{-1} \mathrm{e}^{-z} \mathrm{~d} z \prec \infty
$$

The first term of equation is finite because the integrand is continuous on the finite closed interval $[0,1]$. Also the finiteness of the term $\int_{1}^{\infty} z^{-1} \mathrm{e}^{-z} \mathrm{~d} z$ is immediate because of the inequality $z^{-1} \mathrm{e}^{-z} \leq \mathrm{e}^{-z}, z \geq 1$. 
The finiteness of this expression guarantees the existence of a process corresponding to this measure, this process is $\Gamma(1,1)$. Replacing this measure, the integral equation becomes:

$$
\begin{aligned}
\int_{\Re}\left[1-(1+y z)^{\gamma-1}\right] z v(\mathrm{~d} z) & =\int_{0}^{\infty}\left[1-(1+y z)^{\gamma-1}\right] \mathrm{e}^{-z} \mathrm{~d} z \\
& =\int_{0}^{\infty} \mathrm{e}^{-z} \mathrm{~d} z-\int_{0}^{\infty}(1+y z)^{\gamma-1} \mathrm{e}^{-z} \mathrm{~d} z \\
& =1-\int_{0}^{\infty}(1+y z)^{\gamma-1} \mathrm{e}^{-z} \mathrm{~d} z
\end{aligned}
$$

Now, we can rewrite the remaining integral by using a variable change $U=1+y z$, where $z=\frac{U-1}{y}$, we find

$$
\begin{aligned}
\int_{1}^{\infty} U^{\gamma-1} \mathrm{e}^{-\frac{U}{y}} \mathrm{e}^{\frac{1}{y}} \mathrm{~d} U & =\frac{1}{y} \mathrm{e}^{\frac{1}{y}} \int_{1}^{\infty} U^{\gamma-1} \mathrm{e}^{-\frac{U}{y}} \mathrm{~d} U \\
& =\frac{\mathrm{e}^{\frac{1}{y}}}{y}\left[\int_{0}^{\infty} U^{\gamma-1} \mathrm{e}^{-\frac{U}{y}} \mathrm{~d} U-\int_{0}^{1} U^{\gamma-1} \mathrm{e}^{-\frac{U}{y}} \mathrm{~d} U\right] \\
& =\frac{\mathrm{e}^{\frac{1}{y}}}{y}\left[y^{\gamma} \Gamma(\gamma)-\int_{0}^{1} U^{\gamma-1} \mathrm{e}^{-\frac{U}{y}} \mathrm{~d} U\right]
\end{aligned}
$$

If we take $t=\frac{U}{y}$,

$$
\int_{\mathfrak{R}}\left[1-(1+y z)^{\gamma-1}\right] z v(\mathrm{~d} z)=y^{\gamma-1} \mathrm{e}^{\frac{1}{y}} \Gamma(\gamma)\left[1-P\left(\frac{1}{y}, \gamma\right)\right]
$$

\section{Definition}

We have used a predefined statistical function from the program Matlab to rewrite the integral, the function is called the gamma function which is defined by

$$
P(a, x)=\frac{1}{\Gamma(a)} \int_{1}^{x} t^{a-1} \mathrm{e}^{-t} \mathrm{~d} t
$$

By combining these results, Equation (11) becomes:

$$
\begin{aligned}
& \lambda \sigma_{p}+(\gamma-1) \sigma_{p}^{2} y+\sigma_{r} \sigma_{p} \rho \frac{\gamma}{\alpha}[1-\exp (\alpha(T-t))] \\
& -y^{\gamma-1} \mathrm{e}^{\frac{1}{y}} \Gamma(\gamma)\left[1-P\left(\frac{1}{y}, \gamma\right)\right]=0
\end{aligned}
$$

This equation is highly nonlinear. Therefore, this equation cannot be solved explicitly. With the implementation of a numerical algorithm, we can solve this equation.

\section{Problem Discretization and Parameter Estimates}

In this section, we first estimate the parameters in Equations (2) and (3) of Sec- 
tion 3 by maximum likelihood (ML) method. Then, we show how to implement numerically the portfolio selection model to determine the optimal proportions invested in the cryptocurrencies and gold at different times over the holding period.

Table 1 reports the descriptive statistics (mean, standard deviation, skewness, and kurtosis) of the daily data rate of returns from January 2014 to December 2019 computed from the daily US prices of GOLD, BTC, LTC and DASH obtained from the yahoo finance website.

Skewness and Kurtosis coefficients are calculated to measure the distribution deviation from the symmetry and to measure whether the shape of the distribution deviates from the flattening of the normal distribution. The studied samples reflect two types of asymmetry: A positive asymmetry is estimated for BTC, DASH, and LTC (0.2866), (1.4536), (2.0184) respectively. The three distributions are spread to the right. A negative asymmetry is estimated for gold $(-0.0385)$. Gold distribution is spread to the left. A positive kurtosis is estimated for gold (4.6696), BTC (8.2070), LTC (21.6283), and DASH (12.1697). These coefficients indicate distributions with a flatter peak and thicker ends compared to the normal distribution (leptokurtic distribution).

Figure 1(a) and Figure 1(b) show that the BTC value increases in a huge way. It is also clear that coins are very volatile. The high volatility of coins triggers more chances of generating higher returns. Investors could have higher returns in BTC, LTC, and DASH than in gold. Coins seem to be an attractive investment that can significantly increase the return on a portfolio.

In Figure 1(a) we describe the volatility of daily returns of BTC, LTC and DASH. An analysis of this data shows that volatility tends to fluctuate. From the beginning of 2017, there are slight fluctuations. At the end of 2017, there is a high fluctuation in volatility that goes down by 2018 until the end of the period.

The return series fluctuates around zero. Figure 1(b) illustrates periods of high volatility and periods of low volatility; this may be explained by an effect of volatility clustering, which means that small returns are followed by small returns and the same for large returns.

Graphically, from 2014 to 2017, the return values are almost the same (low fluctuation). In 2017, we have high values. This sudden change in returns can be explained by the fact that the price was too high during this period.

Table 1. Summary of descriptive statistics for daily returns.

\begin{tabular}{ccccc}
\hline & GLD & BTC & LTC & DASH \\
\hline Mean & 0.00003 & -0.0015 & 0.0030 & 0.0033 \\
Stdev & 0.0379 & 0.0388 & 0.0596 & 0.0601 \\
Skewness & -0.0385 & 0.2866 & 2.0184 & 1.4536 \\
Kurtosis & 4.6696 & 8.2070 & 21.6283 & 12.1697 \\
\hline
\end{tabular}




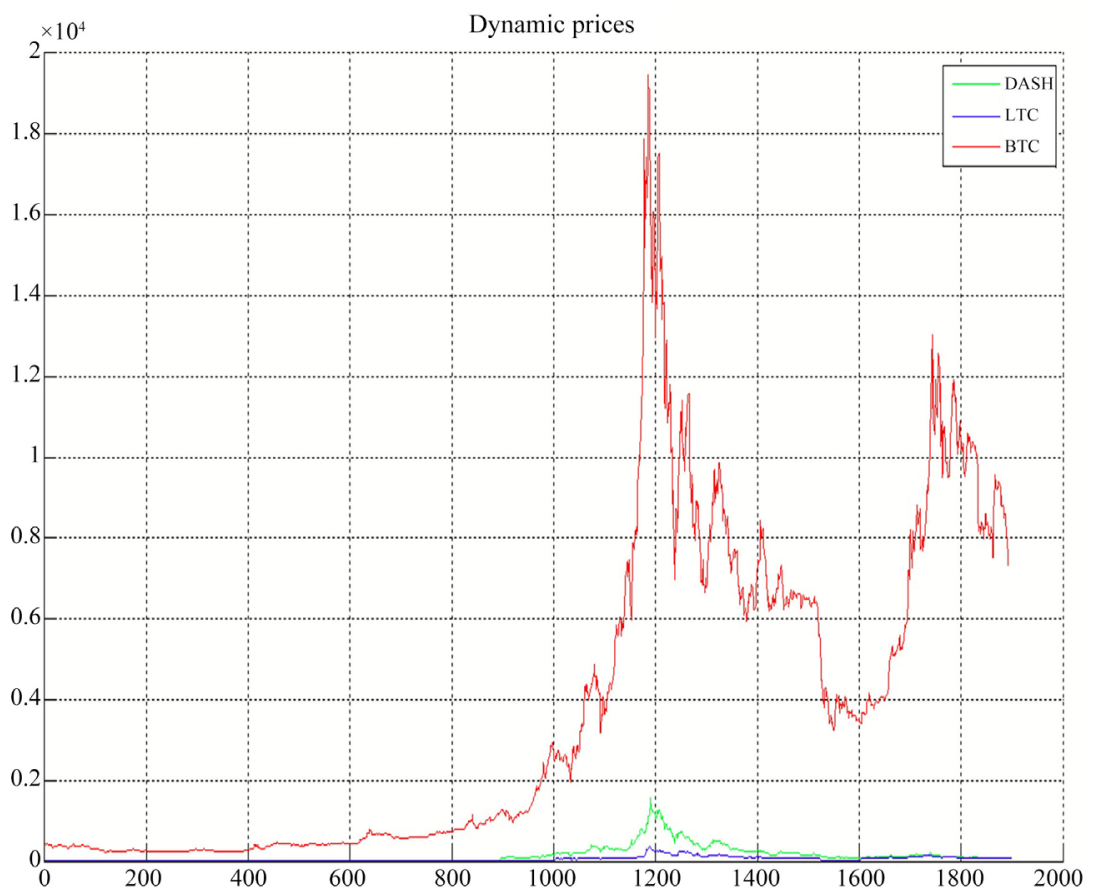

(a)

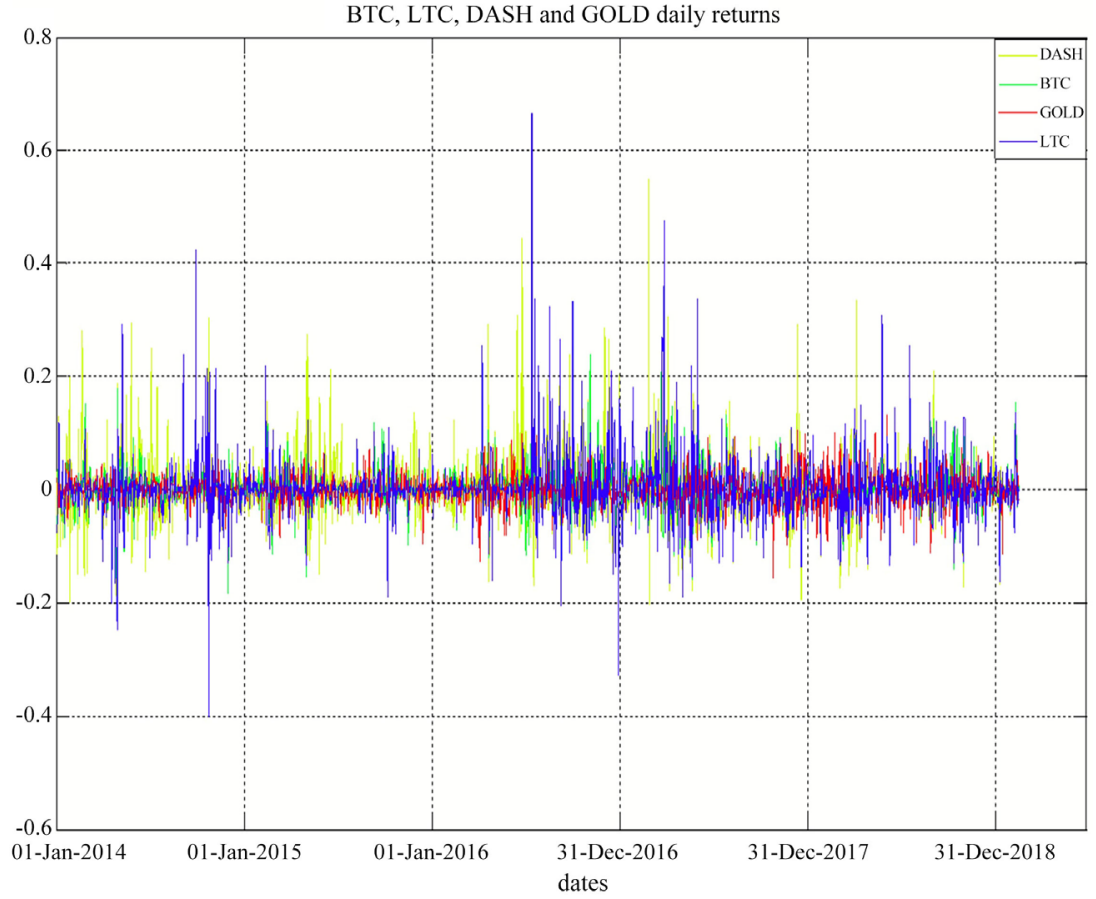

(b)

Figure 1. (a) Price dynamics; (b) Daily returns.

As displayed in Figure 1(a), bitcoin has exhibited extreme fluctuations in its price during 2014 and early 2019. This fluctuation is reflected in the high volatility of cryptocurrency which leads to a huge increase in cryptocurrency returns. We can see an asymmetry between the curves represented by the two Figure 1(a) and Figure 1(b). Indeed, Figure 1(a) shows a sharp increase in the price dy- 
namics of coins in June 2016, in the same period Figure 1(b) shows a huge increase in the yield of crypto. Likewise, Figure 1(a) shows a sharp drop in the price dynamics of coins in September 2017, this decrease in prices leads to a decrease in the yield of cryptocurrency in the same period represented in Figure 1 (b).

To estimate parameters of the Geometric Lévy process, we consider that the return of the cryptocurrencies is generated by a Geometric Brownian motion. Since the integral term does not contain the parameters that must be estimated, this term will disappear by calculating the derivatives for the two parameters $\left(\lambda, \sigma_{p}\right)$. We estimate the parameters in Equations (2) and (3) by using the maximum likelihood (ML) method examined by [27], Analysis of Financial Time Series. Two data sets and a total of 2191 daily price observations, from 1 January 2010 to 31December 2019, are used for the parameter estimation purposes. Both were obtained from the yahoo finance website.

Yahoo Finance application programming interface is a reliable resource of market data and tools that provide financial information including market summaries, historical quotes for any stock ticker symbol, press leases, financial reports and analyse data. It is one of the most important sources of financial data available on the web as it is continuously updated.

We use the gold as a proxy for the short rate $r(t)$ and the BTC, LTC, and DASH for the price $P(t)$. The diffusion process in Equation (2) can be rewritten in discrete form as follows:

$$
r(t+\Delta t)-r(t)=\alpha[\beta-r(t)] \Delta t+\sigma_{r} \varepsilon(r) \sqrt{\Delta t}
$$

where:

$\varepsilon(r)$ is a standard normal deviate, $r(t+\Delta t)-r(t)-\alpha[\beta-r(t)] \Delta t$ is distributed as $N\left(0, \sigma_{r}^{2} \Delta t\right)$

The algorithm of the likelihood function $L\left(\alpha, \beta, \sigma_{r}\right)$, considered as a function of $\alpha, \beta$ and $\sigma_{r}$, can be written as

$$
\begin{aligned}
L\left(\alpha, \beta, \sigma_{r}\right)= & -\frac{n}{2} \ln \left(\sigma_{r}^{2}\right) \Delta t-\frac{n}{2} \ln (2 \pi) \\
& -\frac{\sum_{i=1}^{n}[r(t+\Delta t)-r(t)-\alpha[\beta-r(t)] \Delta t]^{2}}{2 \sigma_{r}^{2} \Delta t}
\end{aligned}
$$

The diffusion process in Equation (3) can be rewritten in discrete form as follows:

$$
\frac{\Delta P(t)}{P(t)}=\left(r+\lambda \sigma_{p}\right) \Delta t+\sigma_{p} \varepsilon(p) \sqrt{\Delta t}
$$

where: $\varepsilon(p)$ is a standard normal deviate.

$$
\ln \left(\frac{\Delta P(t)}{P(t)}\right) \text { is distributed as } N\left(r(t)+\lambda \sigma_{p}-\frac{\sigma_{p}^{2}}{2}, \sigma_{p}\right)
$$

The logarithm of the likelihood function $L\left(\lambda, \sigma_{p}\right)$, considered as a function 
of $\lambda$ and $\sigma_{p}$, can be written as

$$
L\left(\lambda, \sigma_{p}\right)=-\frac{n}{2} \ln \left(\sigma_{p}^{2}\right) \Delta t-\frac{n}{2} \ln (2 \pi)-\frac{\sum_{i=1}^{n}\left[x(t)-\left[r(t)+\lambda \sigma_{p}-\frac{\sigma_{p}^{2}}{2}\right] \Delta t\right]^{2}}{2 \sigma_{p}^{2} \Delta t}
$$

ML estimates are obtained by maximizing Equation (14) for $\alpha, \beta$ and $\sigma_{r}$, Equation (16) for $\left(\lambda_{B}, \sigma_{B}\right),\left(\lambda_{L}, \sigma_{L}\right)$ and $\left(\lambda_{D}, \sigma_{D}\right)$.

The estimates for the nine parameters are as follows:

The results of gold's estimate show that the mean reversion speed and volatility are very low. This indicates that it is a less risky asset. BTC volatility is higher than that of LTC and DASH. The high volatility of BTC explains the high transaction volume and the very high return.

\section{Optimal Portfolio Choices and Behavioral Implications}

We apply the secant method to determine numerically the optimal percentage invested in both assets. The highly risky asset (cryptocurrency) and the less risky asset (gold). The secant method is to approach the function derivative of the first order by a deviated difference. We assume a 5 -year holding period to determine the optimal fraction invested in the two asset classes at each time $t \in[0, T]$ to maximize the expected utility of the terminal wealth. The optimal weights are obtained numerically using Equation (12). The estimated parameters in Equations (2) and (3) using the maximum likelihood estimation method are summarized in Table 2.

Figure 2(a) shows that the optimal percentage invested in BTC, LTC, and DASH starts with $41.52 \%, 41.91 \%$, and $41.48 \%$ respectively and it stabilizes at $50.91 \%, 45.95 \%$, and $46.73 \%$ when gamma is 0.9 . The overtime progression of optimal weights in cryptocurrencies implies that over the medium-term and long-term investors will tend to hold a portfolio uniformly distributed between cryptocurrencies and gold. This behavior means that investors are indifferent between the two types of assets and will end up considering cryptocurrencies as gold a safe investment.

Figures 2(a)-(c) show that optimal weights are more important in cryptocurrencies not only as time goes by but also as the risk aversion coefficient decreases. For Five-year investment horizon, the optimal weight in BTC (LTC) (DASH) goes from $40.96 \%$ to $42.96 \%$ to $50.91 \%$ (40.93\%, $41.97 \%$, 45.95\%) (40.94\%, $42.11 \%$, $46.73 \%$ ) when gamma varies from 0.1 to 0.5 to 0.9 respectively.

Table 2. Parameters estimates.

\begin{tabular}{ccc}
\hline & GOLD & \\
\hline$\hat{\alpha}=0.0666$ & $\hat{\beta}=0.5985$ & $\hat{\sigma}_{r}=0.0390$ \\
BTC & LTC & DASH \\
$\hat{\sigma}_{B}=0.0388$ & $\hat{\sigma}_{L}=0.0183$ & $\hat{\sigma}_{D}=0.0217$ \\
$\hat{\lambda}_{B}=0.2188$ & $\hat{\lambda}_{L}=0.2504$ & $\hat{\lambda}_{D}=0.3278$ \\
\hline
\end{tabular}




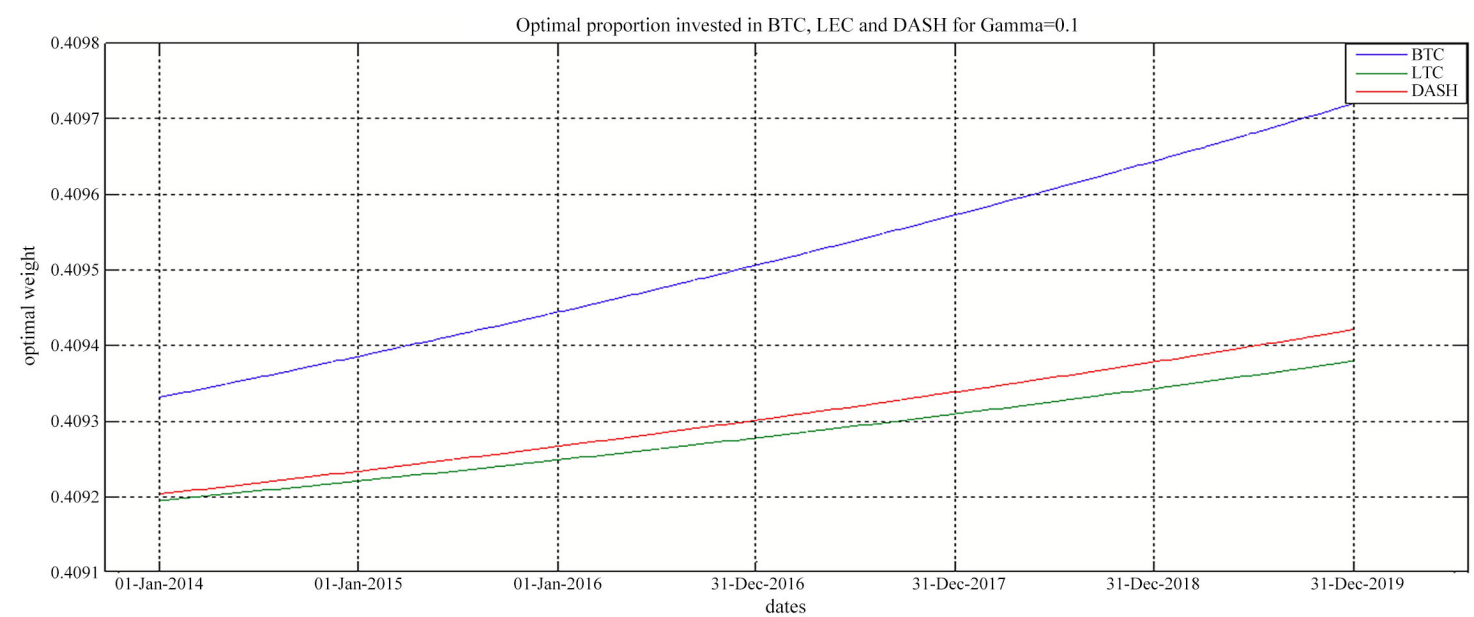

(a)

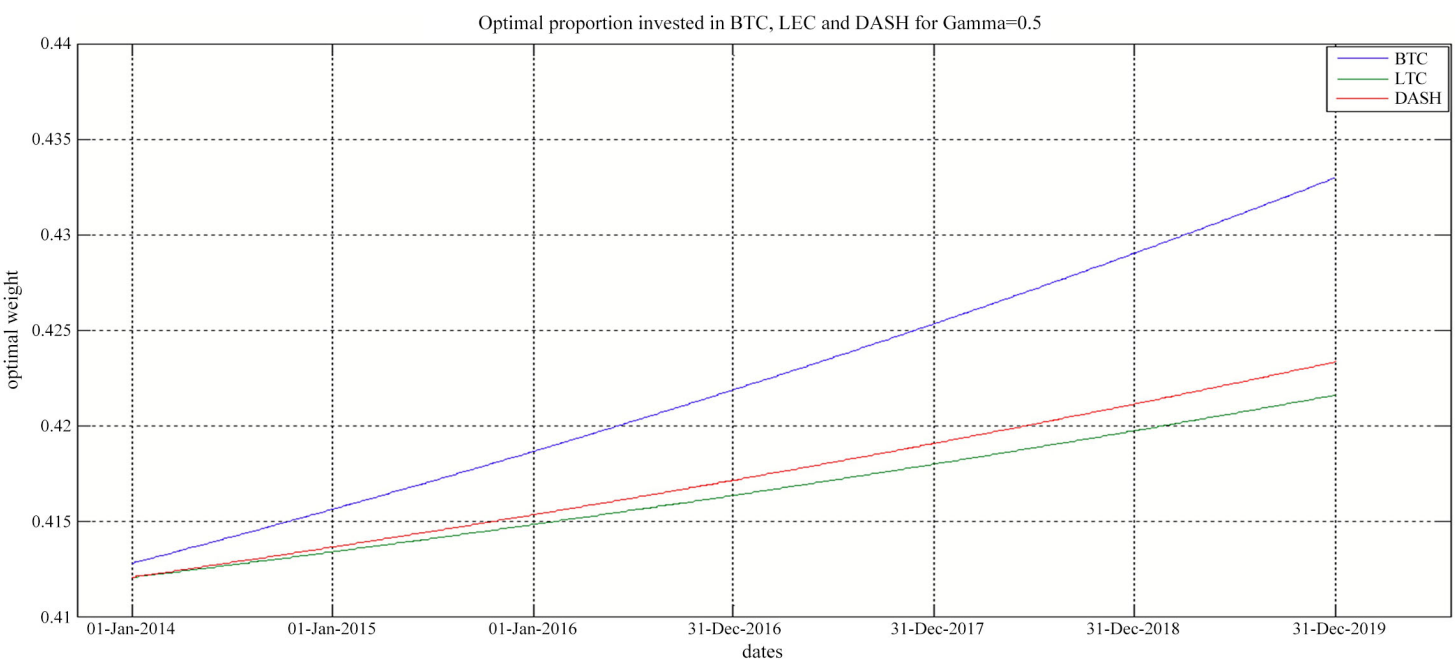

(b)

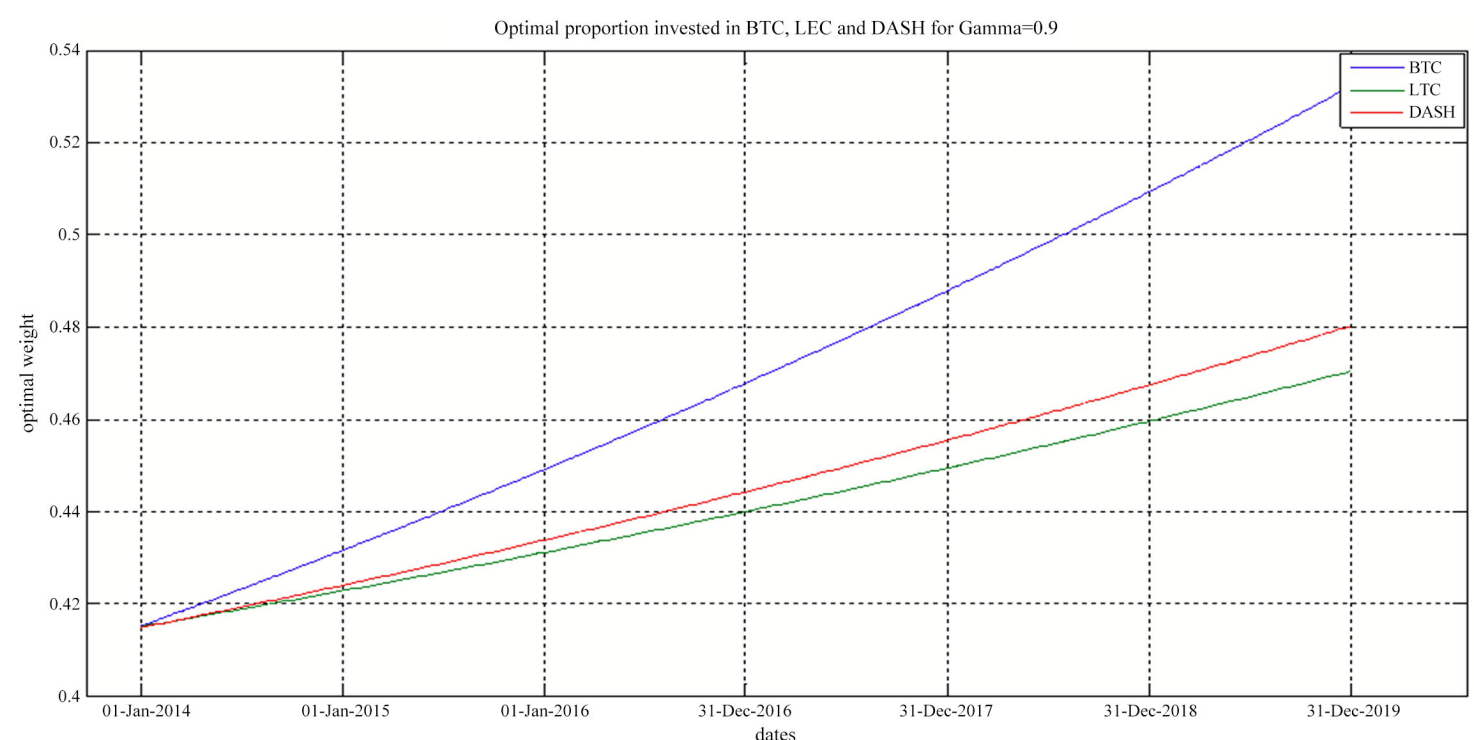

(c)

Figure 2. Optimal proportion invested in BTC, LTC, and DASH. (a) $\gamma=0.1$; (b) $\quad \gamma=0.5$; (c) $\quad \gamma=0.9$. 
These analyses would tend to validate the idea that coins are gradually emerging as a new form of a safe-haven. Gold is a traditional safe-haven. Our analyzes show that BTC, LTC, and DASH could be considered as a substitute for gold since their weights that are taken individually in an optimal investment strategy represent almost half of the proportion invested in Gold. Besides, with the high volatility of coins, there is more chance of generating a better return. An investor could have higher returns in BTC, LTC, and DASH than in Gold. There are more risks, but it is worth for investors (less risk-averse) to generate higher profits. Coins seem to be an attractive investment that can significantly increase the portfolio return. A safe-haven asset is uncorrelated with other asset classes and with the stock market indices. Evan Kuo said, "There have been almost 10 years of data suggesting that the BTC has virtually no exposure to the risk of precious metals, commodities, stocks, bonds, currencies...”. Having an uncorrelated asset can be a good way to balance an investment portfolio against other safe assets. User confidence in BTC is growing day by day and allows us to confirm better this opinion and to build BTC as a refuge in all circumstances. Our results show that the investor's wealth is optimally distributed in equal proportions in the two asset classes and thus BTC, LTC, and Dash seem to be attractive investments for investors and are emerging as a new form of a safe investment.

\section{Conclusion}

In recent decades, cryptocurrencies have been one of the most important financial innovations. They have drawn a growing number of critics and supporters. To analyze cryptocurrencies as assets, we took a stochastic and dynamic modeling approach. In this study, we have dealt with an optimal portfolio model in continuous time over an infinite horizon and in a risky environment to show whether cryptocurrencies such as BTC, LTC, and DASH can replace GOLD in a portfolio of investment or not. Due to the high volatility of cryptocurrency prices, investment portfolios must be reviewed, evaluated in real time and regularly adjusted. For this reason, we worked in a dynamic and stochastic context. The closed form solution of the optimal portfolio is obtained for an investor with a CRRA utility function. Indeed, using daily data, we concluded that cryptocurrencies represent a good substitute for gold in an intertemporal investment portfolio and therefore they represent a new form of safe haven. However, the oldest and the most classic safe haven was gold. BTC, LTC and DASH are emerging as a new form of safe haven that plays an important role in the investment portfolio. Moreover, an investor can replace gold with BTC, LTC or DASH in an investment portfolio and enjoys a high return. This justifies the fact that crypto currencies are becoming a more attractive investment for investors. In this case, a cryptocurrency can also be a safe haven. You can even escape with your codes to the other side of the world, so without losing your Bitcoins or the like. In the long run, like GOLD, the rating of your cryptocurrency portfolio will tend to go up, even if you need to sell it at any time. This has led us to say that BTC, LTC 
and DASH become like digital gold. This analysis provides a clear answer to the question "Can Bitcoin replace gold in an investment portfolio?" that was posed by [3]. Our analysis is carried out on a medium-term investment portfolio (for a period of 5 years from 2014 to 2019), to confirm the idea that BTC becomes a new form of safe-haven. We assert that the BTC and all coins such as LTC and DASH, which have a high transaction volume, behave like digital Gold.

The results presented in this paper concern only the coin data under JumpDiffusion model in which we only assume jump sizes that are larger than -1 , so we may have jumps in both directions but negative jumps cannot be too large. However, for further works, we can change the model of the risky asset to include larger negative jumps.

\section{Conflicts of Interest}

The authors declare no conflicts of interest regarding the publication of this paper.

\section{References}

[1] Yermack, D. (2013) Is Bitcoin a Real Currency? An Economic Appraisal. Working Paper No. w19747, National Bureau of Economic Research, Cambridge. http://www.nber.org/papers/w19747.pdf https://doi.org/10.3386/w19747

[2] Popper, N. (2015) Digital Gold: The Untold Story of Bitcoin. Penguim, London.

[3] Irene, H. and Perry, S. (2018) Can Bitcoin Replace Gold in an Investment Portfolio? Journal of Risk and Financial Management, 11, 48. https://doi.org/10.3390/jrfm11030048

[4] Klein, T., Thu, H.P. and Walther, T. (2018) Bitcoin Is Not the New Gold-A Comparison of Volatility, Correlation, and Portfolio Performance. International Review of Financial Analysis, 59, 105-116. https://doi.org/10.1016/j.irfa.2018.07.010

[5] Pradipta, K.S. (2017) Bitcoin as Digital Money: Its Growth and Future Sustainability. Theoretical and Applied Economics, 24, 53-64.

[6] Corbet, S., Lucey, B. and Yarovaya, L. (2018) Dates Tamping the Bitcoin and Ethereum Bubbles. Finance Research Letters, 26, 81-88. https://doi.org/10.1016/j.frl.2017.12.006

[7] Dwyer, G.P. (2015) The Economics of Bitcoin and Similar Private Digital Currencies. Journal of Financial Stability, 17, 81-91. https://doi.org/10.1016/j.jfs.2014.11.006

[8] Chaim, P. and Laurini, M.P. (2018) Volatility and Return Jumps in Bitcoin. Economics Letters, 173, 158-163. https://doi.org/10.1016/j.econlet.2018.10.011

[9] Smales, L.A. (2018) Bitcoin as a Safe Haven: Is It Even Worth Considering? Finance Research Letters, 30, 385-393. https://doi.org/10.1016/j.frl.2018.11.002

[10] Corbet, et al. (2018) Exploring the Dynamic Relationships between Cryptocurrencies and Other Financial Assets. Economics Letters, 165, 28-34. https://doi.org/10.1016/j.econlet.2018.01.004

[11] Dyhrberg, A.H. (2015) Hedging Capabilities of Bitcoin. Is It the Virtual Gold? Finance Research Letters, 16, 139-144. https://doi.org/10.1016/j.frl.2015.10.025

[12] Bouoiyour, J. and Selmi, R. (2017) Are Trump and Bitcoin Good Partners? Working 
Paper. https://ideas.repec.org/p/hal/wpaper/hal-01480031.html

[13] Eisl, A., Gasser, S.M. and Weinmayer, K. (2015) Caveat Emptor: Does Bitcoin Improve Portfolio Diversification? https://doi.org/10.2139/ssrn.2408997

http://papers.ssrn.com/sol3/papers.cfm?abstract id=2408997

[14] Molnár, P., Vagstad, K. and Valstad, O.C.A. (2015) A Bit Risky? A Comparison between Bitcoin and Other Assets Using an Intraday Value at Risk Approach. Working Paper. http://www.diva-portal.org/smash/get/diva2:742882/fulltext01.pdf

[15] Briere, M., Oosterlinck, K. and Szafarz, A. (2015) Virtual Currency, Tangible Return: Portfolio Diversification with Bitcoin. Journal of Asset Management, 16, 365 373. https://doi.org/10.1057/jam.2015.5

[16] Popper, N. (2015, April 29) Can Bitcoin Conquer Argentina? The New York Times.

[17] Popper, N. (2015) Digital Gold: Bitcoin and the Inside Story of Misfits and Millionaires Trying to Reinvent Money. HarperCollins, New York.

[18] Wang, G.-J., et al. (2020) Volatility Connectedness in the Cryptocurrency Market: Is Bitcoina Dominant Cryptocurrency? International Review of Financial Analysis, 60, 98-114. https://doi.org/10.1016/j.irfa.2018.08.012

[19] Dyhrberg, A.H. (2016) Bitcoin, Gold and the Dollar-A GARCH Volatility Analysis. Finance Research Letters, 16, 85-92. https://doi.org/10.1016/j.frl.2015.10.008

[20] Bouri, E., Molnar, P., Azzi, G., Roubaud, D. and Hagfors, L.I. (2017) On the Hedge and Safe Haven Properties of Bitcoin: Is It Really More than a Diversifier? Finance Research Letters, 20, 192-198. https://doi.org/10.1016/j.frl.2016.09.025

[21] Baur, D.G., Hong, K. and Lee, A.D. (2018) Bitcoin: Medium of Exchange or Speculative Assets? Journal of International Financial Markets, Institutions, and Money, 54, 177-189. https://doi.org/10.1016/j.intfin.2017.12.004

[22] Bouri, E., Gil-Alana, L.A., Gupta, R. and Roubaud, D. (2016) Modelling Long Memory Volatility in the Bitcoin Market: Evidence of Persistence and Structural Breaks. Working Paper No. 2016-54, University of Pretoria, Pretoria. http://www.up.ac.za/media/shared/61/WP/wp 2016 54.zp92472.pdf

[23] Copeland, T.E., Fred Weston, J. and Shastri, K. (2005) Financial Theory and Corporate Policy. 15th Edition, Addison-Wesley, Boston.

[24] Campbell, J.Y. and Viceira, L.M. (2002) Strategic Asset Allocation: Portfolio Choice for Long-Term Investors. Oxford University Press, Oxford.

https://doi.org/10.1093/0198296940.001.0001

[25] Øksendal, B. and Sulem, A. (2007) Applied Stochastic Control of Jump Diffusions. Second Edition, Springer, Berlin. https://doi.org/10.1007/978-3-540-69826-5

[26] Fatma, C. and Fathi, A. (2016) Capital Adequacy and Risk Management in Banking Industry. Applied Stochastic Models in Business and Industry, 32, 113-123. https://doi.org/10.1002/asmb.2127

[27] Tsay, R.S. (2005) Analysis of Financial Time Series. 2nd Edition, John Wiley \& Sons, New York. https://doi.org/10.1002/0471746193 